$\xi$

\title{
Velocity Slip and Thermal Jump on Maxwell Fluid with Non-Fourier Cattaneo-Christov Heat Flux Using SRM Solutions
}

\author{
K. Gangadhar ${ }^{1}$, K. V. Ramana ${ }^{2}$ and B. Rushi Kumar ${ }^{3 *}$ \\ ${ }^{l}$ Department of Mathematics, Acharya Nagarjuna University, Ongole, Andhra Pradesh-523001,India \\ ${ }^{2}$ Deparment of Mathematics, NRI Institute of Technology, Guntur, Andhra Pradesh-522438, India \\ ${ }^{3}$ Department of Mathematics, School of Advanced Sciences, VIT, Vellore-632014,TN, India \\ *Corresponding author E-mail: rushikumar@vit.ac.in
}

\begin{abstract}
The influence of the heat transfer within a boundary layer flow and magneto hydro dynamic slip flow of a Maxwell fluid over a stretching cylinder is analyzed and discussed in the present article. The effects of viscous dissipation and thermal jump are assumed. The procedure of heat transfer through hypothesis of Cattaneo-Christov heat flux is considered. We converted non-linear partial differential equations for mass, momentum and energy into a system of coupled highly non linear ordinary differential equations with proper boundary conditions by the help of suitable similarity transformations. The succeeding ordinary differential equations are solved by using Spectral relaxation technique. The solution is obtained in zero curvature parameter as well as non-zero curvature parameter. i.e. for flow above a flat plate and flow above a cylinder. The flow and heat transfer attributes are witnessed to be encouraged in an elaborate mode by Prandtl number, thermal jump parameter, thermal relaxation parameter, Deborah number, slip velocity parameter, Eckert number and the magnetic parameter. Our findings reveal that one of the possible ways to decrease the Deborah number by boosting fluid velocity. It is also perceived that in the case of flow over a stretching cylinder, the momentum boundary layer thickness and the velocity of the fluid increases. Furthermore, an increase in slip velocity factor reduces the magnitude of skin friction.
\end{abstract}

Keywords: Cattaneo-Christov heat flux model, Stretching cylinder, Temperature Jump, MHD, Velocity slip.

\section{Introduction}

The performance and attributes of non-Newtonian fluids is an important subject for researchers as well as scientists due to plentiful applications of non-Newtonian fluids in petroleum products, pharmaceuticals, paper construction, crystal growth, polymer sheet, the coating of wires and so on. A single constitutive relationship is not enough to mention the physical attributes of nonNewtonian fluids. Relaxation time is only depending factor of Maxwell fluid. Due to this consequence, Maxwell fluid over with various geometries was observing by researchers. The short review of Maxwell fluid above a cylinder was given by [1-3].

The investigation of non-Newtonian fluids affected by the magnetic field is proposing the concentration of its multidisciplinary applications for example, purification of crude oil, MHD power controllers, electromagnetic propulsion and nuclear reactors. Due to this, Hayat [4] and Rashidi [5] shown that the volume fraction of nanoparticles, mixed convection parameter improves the wall skin friction coefficient and also displayed the investigative as well as numerical solutions of the flow over a cylinder with diverse flow properties. Shateyi [6] mentioned that fluid temperature increases by an increase of Eckert number and thermal radiation. Shateyi [6] also demonstrated thermophoresis effects and Maxwell fluid flow with chemical reaction over a stretching surface. By the frictional heating energy of heat is stored in the liquid. Consequently, the influence of improving Eckert number is increases the fluid temperature. Likewise, rise in the thermal radiation parameter values signifies the attractive of radiation in the boundary layer and accordingly rises temperature profiles in the area of thermal boundary layer.

In micro/nanosystems a most important task played by slip flow such are hard disk drive, micropumps, nozzles and microvalves. Sajid et al. [7] examined that the the slip influence on the Maxwell fluid flow over a surface of stretching. Sajid et al. [7] and Cai [8] explored the joint effect of temperature jump and velocity slip on a boundary layer flow over a flat plate. The effect of velocity slip and temperature jump on a boundary layer flow of pseudo-plastic power law fluid towards a moving permeable surface in occurrence of magnetic field utilizing bvp4c with MATLAB was investigated by Xinnui et al. [9]. They fulfilled that the skin friction coefficient and heat transfer rates diminish with the rising values of thermal slip and jump parameters. The temperature jump and velocity slip influences on unsteady boundary layer flow from a stretching permeable surface utilizing rational Bernstein collocation method was presented by Hosseini et al. [10]. By finite difference method of implicit functions the slip effect and convective boundary condition on magneto hydro dynamic boundary layer flow of a nanofluid over a porous nonlinear stretching shrinking sheet have been numerically investigated by Daniel et al. [11].

By a lot of uses of Heat transfer, it becomes a major research area to upcoming researchers. Heat transfer uses in nuclear reactors, materials processing, energy production and rocket thermal ablation etc. A classical approach of simulation of thermal conduction heat transfer is the Fourier law of heat conduction [12]. This model reduces heat conservative to parabolic energy equation which exhibits that the medium under observation encounters an initial disturbance which is the major drawback of this model. So to reduce disturbance, Cattaneo [13] modified the Fourier's law by 
including a relaxation time. This model further refined by Christov [14] that a structure in diverse formulation for the MaxwellCattaneo model with finite speed heat conduction. An equation of the Maxwell-Cattaneo was numerically explained by OstojaStarzewski [15] with a material time derivative for heat flux. The uniqueness and stability of solutions for incompressible fluid acquired by heat flux model of Cattaneo-Christov were explored by Zampoli and Tibullo [16]. By using the Cattaneo-Christov model, Straughan [17] examined numerically an incompressible thermal convection flows. If the Cattaneo number is very large then the thermal relaxation coefficient is more important which is found by Straughan [17]. Straughan [17] also found that the convection phenomena transform from stationary to oscillatory convection which is having narrower cells. Haddad [18] conducted an investigation of thermal unsteadiness incorporating fluid inertia utilizing heat flux model through a Brinkman porous medium. The structural steadiness and uniqueness equations of heat flux which are given by Cattaneo-Christov were addressed by Ciarletta and Straughan [19]. They also demonstrate that the relaxation time is only depending factor of solution to a backward in time problems. Al-Qahtani and Yilbas [20] presented a closed form solution by using the Laplace transform method for Cattaneo and stress equations. By using vertical and horizontal gradients, the influences of thermal relaxation in the Cattaneo-Maxwell equations were examined by Papanicolaou et al. [21]. The boundary layer flow of Maxwell fluids from a stretching sheet by using the CattaneoChristov heat flux model was studied by Han et al. [22]. The nonFourier convection in rotary Maxwell fluid flow was numerically and systematically examined by Mustafa [23]. He offered both numerical and analytical solutions and displayed those results are in excellent manner. In place of parabolic Fourier law, CattaneoChristov heat flux model used in oscillatory convection flow to augment the opportunity of oscillatory convection in a classic Bernard problem was analyzed by Bissell [24]. The chemically reactive boundary layer flow of upper-convected Maxwell fluid with the effect of Cattaneo-Christov heat flux model utilizing HAM technique was examined by Khan et al. [25]. Raju et al. [26] analyzed the influence of MHD on a boundary layer flow of a Maxwell non-Fourier fluid over a cylinder under CattaneoChristov heat flux model utilizing Runge-Kutta based shooting procedure. They accomplished that in impermeable flow over a cylinder case, the skin friction coefficient is high when compared with the permeable flow over a cylinder case. Shahid et al. [27] examined that the model of Cattaneo-Christov heat flux influence on radiative Maxwell viscoelastic magnetic flow over stretching permeable sheet. Khan et al. [28], investigated, the hyperbolic heat flux on upper-convected Maxwell micro polar fluid over a semi infinite stretching surface.

Reasonably some studies of magneto hydro dynamic nonNewtonian slip flow and convective heat flows for have occurred with a non-Fourier formulation for thermal conduction. The purpose of this article is to analyze the hydrodynamic forced convective heat transfer in a boundary layer slip flow of Maxwell fluid from a stretching cylinder with a model of non-Fourier CattaneoChristov heat flux. Moreover the joint impacts of viscous dissipation and thermal jump are taken into consideration. This flow problem is applicable to polymeric materials processing operations in which thermal loading may be of the non-Fourier type [29-32]. By using the graphs that the physical impact of the emerging parameters on temperature, velocity, Nusselt number and skin friction coefficient is elaborated. The numerical results obtained by using SRM technique are compared with existing published results.

\section{Mathematical Formulation}

A steady and axisymmetric boundary layer flow of an incompressible slip flow of Maxwell fluid over stretching cylinder of radius $\mathrm{R}$ over a stretching cylinder is considered. The Maxwell fluid is being moved along the axial $\mathrm{x}$ direction and radial coordinate, $\mathrm{r}$, is perpendicular to the cylinder axial. Magneto hydrodynamic flow analysis is presented along Cattaneo-Christov heat flux and viscous dissipation. Cylindrical coordinates are selected that $\mathrm{x}$-axis is along the axial direction of the cylinder and $\mathrm{r}$-axis is perpendicular to cylinder axial. As shown in Figure 1, stretching velocity of the cylinder is organized by applying two forces that are equal in magnitude, opposite in direction such that origin is kept constant.

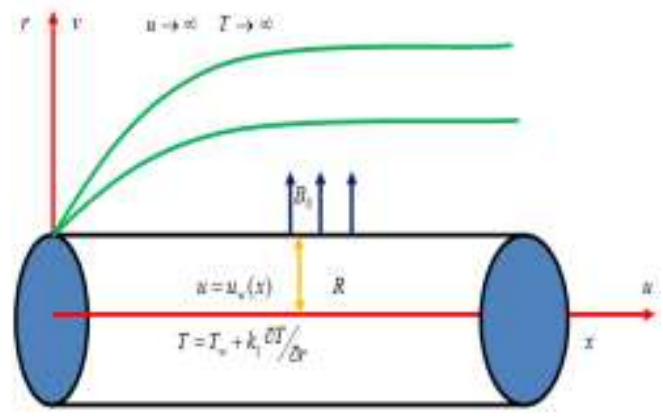

Fig. 1: Physical model and coordinate system

Apply a uniform magnetic field of strength $B_{0}$ in the radial direction. Here the obtained magnetic field is to be ignored because magnetic Reynolds number is acknowledged to be smaller. The surface and ambient temperatures are taken as $T_{w}$ and $T_{\infty}$, respectively. The conversation of mass, momentum and energy equations takes the following form by applying the assumptions.

$\frac{\partial(r u)}{\partial x}+\frac{\partial(r v)}{\partial y}=0$

$$
\begin{aligned}
\left(u \frac{\partial u}{\partial x}+v \frac{\partial u}{\partial r}\right)+\beta_{1}\left(u^{2} \frac{\partial^{2} u}{\partial x^{2}}+2 u v\right. & \left.\frac{\partial^{2} u}{\partial x \partial r}+v^{2} \frac{\partial^{2} u}{\partial r^{2}}\right) \\
& =v \frac{\partial^{2} u}{\partial r^{2}}+\frac{v}{r} \frac{\partial u}{\partial r}-\frac{\sigma B_{0}{ }^{2}}{\rho} u
\end{aligned}
$$

$$
\left(\begin{array}{l}
\left.u \frac{\partial T}{\partial x}+v \frac{\partial T}{\partial r}\right)+\beta_{2}\left(\begin{array}{l}
u^{2} \frac{\partial^{2} T}{\partial x^{2}}+2 u v \frac{\partial^{2} T}{\partial x \partial r} \\
+v^{2} \frac{\partial^{2} T}{\partial r^{2}}+\frac{\partial T}{\partial x}\left(u \frac{\partial T}{\partial x}+v \frac{\partial T}{\partial r}\right) \\
+\frac{\partial T}{\partial r}\left(u \frac{\partial T}{\partial x}+v \frac{\partial T}{\partial r}\right)
\end{array}\right) \\
=\frac{k}{\rho c_{p}}\left(\frac{\partial^{2} T}{\partial r^{2}}+\frac{1}{r} \frac{\partial T}{\partial r}\right)+\frac{v}{c_{p}}\left(\frac{\partial u}{\partial r}\right)^{2}
\end{array}\right.
$$

The consequent boundary conditions for the present problem are imposed as below.

$$
\begin{aligned}
& u=u_{w}(x)+L_{1}\left(\frac{\partial u}{\partial r}\right), \quad v=0, \quad T=T_{w}++k_{1}\left(\frac{\partial T}{\partial r}\right) \text { at } r=R, \\
& u \rightarrow 0, T \rightarrow T_{\infty} \text { at } r \rightarrow \infty
\end{aligned}
$$

In above expressions $\mathrm{u}$ represents velocity component in the axial direction and $\mathrm{v}$ represents velocity component in the radial direction, $B_{0}$ represents the magnetic field, $\sigma$ is the electrical conductivity, $\beta_{1}$ represents the relaxation time and $\beta_{2}$ represents the thermal relaxation time, $u_{w}=U_{0} x / l$ represents stretching velocity $\mathrm{R}$ represents radius of cylinder, $v$ represents the kinematic viscosity, $\rho$ represents the density, $k$ represents the thermal conductivity, $T$ represents the cylinder surface temperature, $l$ represents 
the characteristics length, $L_{1}$ represents the slip coefficient and $k_{1}$ represents the jump coefficient, $c_{p}$ represents the specific heat at constant pressure. The following dimensionless quantities transform the governing equations.

$\zeta=\sqrt{\frac{U_{0}}{v l}}\left(\frac{r^{2}-R^{2}}{2 R}\right), \quad \psi=\sqrt{U_{w} v x} R f(\zeta)$

$u=\frac{U_{0} x}{l} f^{\prime}(\zeta)$

$v=-\sqrt{\frac{v U_{0}}{l}} \frac{R}{r} f(\zeta), \theta(\zeta)=\frac{T-T_{w}}{T_{\infty}-T_{w}}$,

$u=\frac{\partial \psi}{\partial y}, \quad v=-\frac{\partial \psi}{\partial x}$

We observe that the continuity equation (1) is automatically fulfilled by Eq. (5). Equations (2) and (3) are altered as

$$
\begin{array}{r}
(1+2 \gamma \zeta) f^{\prime \prime \prime}(\zeta)+2 \gamma f^{\prime \prime}(\zeta)-M f^{\prime}(\zeta)+f(\zeta) f^{\prime \prime}(\zeta)-f^{\prime 2}(\zeta) \\
-\lambda_{1}\left(f^{2}(\zeta) f^{\prime \prime \prime}(\zeta)-2 f(\zeta) f^{\prime \prime}(\zeta)\right)=0 \\
(1+2 \gamma \zeta) \theta^{\prime \prime}(\zeta)+2 \gamma \theta^{\prime}(\zeta)+\operatorname{Pr} f(\zeta) \theta^{\prime}(\zeta) \\
-\operatorname{Pr} \lambda_{2}\left(f^{2}(\zeta) \theta^{\prime \prime}(\zeta)-f(\zeta) \theta^{\prime}(\zeta) f^{\prime}(\zeta)\right)+\operatorname{Pr} E c f^{\prime \prime 2}(\zeta)=0
\end{array}
$$

and the boundary constraints changes as

$$
\begin{aligned}
& f(\zeta)=0, f^{\prime}(\zeta)=1+\alpha_{1} f^{\prime \prime}(\zeta), \theta(\zeta)=1+\alpha_{2} \theta^{\prime}(\zeta) \text { at } \zeta=0 \\
& f^{\prime}(\zeta)=0, \theta(\zeta)=0 \quad \text { as } \quad \zeta \rightarrow \infty
\end{aligned}
$$

Where $\gamma$ represents the curvature parameter, $\lambda_{1}, \lambda_{2}$ represents the Deborah numbers in terms of velocities time and thermal relaxation parameter respectively, Pr represents the Prandtl number, Ec represents the Eckert number, $M$ represents the magnetic field parameter, $\alpha_{1}$ represents the velocity slip factor, $\alpha_{2}$ represents the temperature jump factor which are specified by

$$
\begin{aligned}
& \gamma=\left(\frac{v l}{U_{0} R^{2}}\right)^{\frac{1}{2}}, \quad \operatorname{Pr}=\frac{\mu c_{p}}{k}, \quad \lambda_{1}=\frac{\beta_{1} U_{0}}{l}, \quad \lambda_{2}=\frac{\beta_{2} U_{0}}{l} \\
& M=\frac{\sigma B_{0}{ }^{2}}{a \rho}, \quad E c=\frac{U_{w}^{2}}{c_{p}\left(T_{w}-T_{\infty}\right)}, \alpha_{1}=L_{1} \sqrt{\frac{U_{0}}{v l}}, \quad \alpha_{2}=k_{1} \sqrt{\frac{U_{0}}{v l}}
\end{aligned}
$$

For engineering attention, we also establish the physical quantities of the system of reduced local Nusselt number and shear stress coefficient are $N u_{x}$ and $C_{f}$, respectively given by

$\operatorname{Re}_{x}^{\frac{-1}{2}} N u_{x}=-\theta^{\prime}(0)$

$$
\operatorname{Re}_{x}^{\frac{1}{2}} C f_{x}=f^{\prime \prime}(0)
$$

With $\operatorname{Re}_{x}=\frac{U_{w} x}{v}$ is a Reynolds number.

\section{Solution of the Problem}

With a specific end goal, the Spectral Relaxation Method (SRM) given by Motsa and Makukula [33] and Kameswaran et al. [34] is used to deal with the conditions (6)-(7) subject to the boundary conditions (8) and (9). The systems of equations are altered by Gauss- Seidel method. The iteration scheme obtained by SRM is

$$
\begin{aligned}
& f_{r+1}^{\prime}=p_{r}, f_{r+1}(0)=0 \\
& \left(1+2 \gamma \zeta-\lambda_{1} f_{r+1}^{2}\right) p_{r+1}^{\prime \prime}+\left(2 \gamma+f_{r+1}+2 \lambda_{1} f_{r+1}\right) p_{r+1}^{\prime}-(M n) p_{r+1}=p_{r+1}^{2} \\
& \left(1+2 \gamma \zeta-\operatorname{Pr} \lambda_{2} f_{r+1}^{2}\right) \theta_{r+1}^{\prime \prime}+\left(2 \gamma+\operatorname{Pr} f_{r+1}+\operatorname{Pr} \lambda_{2} f_{r+1} p_{r+1}\right) \theta_{r+1}^{\prime} \\
& =-\operatorname{Pr} E c p_{r+1}^{\prime 2}
\end{aligned}
$$

By the iteration scheme the boundary conditions changes as

$$
\begin{aligned}
& p_{r+1}(0)=1+\alpha_{2} p_{r+1}^{\prime}(0), \theta_{r+1}(0)=1+\alpha_{3} \theta_{r+1}^{\prime}(0) \\
& p_{r+1}(\infty)=0, \theta_{r+1}(\infty)=0
\end{aligned}
$$

With a specific end goal, we apply the Chebyshev spectral collocation technique to comprehend the decoupled equations (13) (15). In the process of spectral relaxation method, the domain [0, L] transforms as $[-1,1]$ with the substitution $\eta=L(\xi+1) / 2$. Where $L$ represents the boundary conditions at $\infty$. The necessary consideration behind the spectral collocation technique is the beginning of a differentiation matrix $\mathcal{D}$ which is utilized to expect the derivatives of the unidentified variables at the collocation points and the matrix vector product of form

$$
\frac{\partial f_{r+1}}{\partial \eta}=\sum_{k=0}^{N} D_{l k} f_{r}\left(\xi_{k}\right)=D f_{r}, l=0,1,2, \ldots \ldots \ldots . . . N
$$

Where $N+1$ represents number of collocation points (grid points), $D=2 \mathcal{D} / L, \quad=\quad$ and $f=\left[f\left(\xi_{0}\right), f\left(\xi_{1}\right), f\left(\xi_{2}\right), \ldots \ldots \ldots f\left(\xi_{N}\right)\right]^{T}$ is represents the vector function at the collocation points. The derivatives of highest order are acquired in powers of $D$, that is,

$$
f_{r}^{(p)}=D^{p} f_{r}
$$

Where $p$ denotes the order of the derivative. By implementing the spectral relaxation technique to equations (25)- (27), we get

$$
\begin{aligned}
& A_{1} f_{r+1}=B_{1}, f_{r+1}\left(\xi_{N}\right)=0 \\
& A_{2} p_{r+1}=B_{2}, \alpha_{1} \sum_{k=0}^{\bar{N}} D_{\bar{N} k} p_{r+1}\left(\xi_{k}\right)-p_{r+1}\left(\xi_{k}\right)=-1, p_{r+1}\left(\xi_{0}\right)=0 \\
& A_{3} \theta_{r+1}=B_{3}, \alpha_{2} \sum_{k=0}^{\bar{N}} D_{\bar{N} k} \theta_{r+1}\left(\xi_{k}\right)-\theta_{r+1}\left(\xi_{k}\right)=-1, \theta_{r+1}\left(\xi_{0}\right)=0
\end{aligned}
$$

Here,

$A_{1}=D, B_{1}=p_{r+1}$

$A_{2}=\left(1+2 \gamma \zeta-\lambda_{1} f_{r+1}^{2}\right) D^{2}+\operatorname{diag}\left(2 \gamma+f_{r+1}+2 \lambda_{1} f_{r+1}\right) D-M n I$

$B_{2}=p_{r+1}^{2}$

$A_{3}=\left(1+2 \gamma \zeta-\operatorname{Pr} \lambda_{2} f_{r+1}^{2}\right) D^{2}+\operatorname{diag}\left(2 \gamma+\operatorname{Pr} f_{r+1}+\operatorname{Pr} \lambda_{2} f_{r+1} p_{r+1}\right) D$

$B_{3}=-\operatorname{Pr} E c p_{r+1}^{\prime 2}$ 
In equations (23)-(25), an identity matrix represented by $I$, and represents diagonal matrix represented by [] are of size $(N+1) \times(N+1)$ where $N$ represents the number of grid points, at the grid points the values of the functions $f, p$ and $\theta$ are $f_{r}, p_{r}$ and $\theta_{r}$ respectively where the subscript $\mathrm{r}$ represents an iteration number.

To start the SRM technique for equalities (16)-(17), assumed initial conditions are

$$
f_{0}(\eta)=\frac{1}{1+\alpha_{1}}\left(1-e^{-\eta}\right), p_{0}(\eta)=\frac{1}{1+\alpha_{1}} e^{-\eta}, \theta_{0}(\eta)=\frac{1}{1+\alpha_{2}} e^{-\eta}
$$

Which are arbitrarily selected functions that are gratify the boundary conditions. To achieve the convergence we are repeating the above process. The convergence of the SRM technique is represented in vocabulary of the infinity norm as

$$
E r=\operatorname{Max}\left(\left\|f_{r+1}-f_{r}\right\|,\left\|p_{r+1}-p_{r}\right\|,\left\|\theta_{r+1}-\theta_{r}\right\|\right)
$$

Exactness of the method is familiar by raising the number of collocation points $N$ until the solutions are reliable and moreover raises do not modify the assessment of the results.

Validation of Numerical Solution: To evaluate the precision of numerical method, we have good concurrence for the numerical values of Nusselt number $\theta^{\prime}(0)$ in the lack of viscous dissipation, MHD, slip influences and Cattaneo-Christov heat flux in the case of Newtonian fluid i.e. $\mu=E c=\lambda_{1}=\lambda_{2}=\alpha_{1}=\alpha_{2}=0$ in opposition to Prandtl number values with those of Grubka and Bobba [35], Ali [36], Chen [37] and Ishak [38], which is presented in table 1 . This ensures that our numerical scheme is a certainty and it can be used for further calculation of results.

\section{Results and Discussion}

In this segment complete numerical calculations are accessible for different parameters on temperature, transverse velocity, Nusselt number and skin friction coefficients profiles. By using the graphical representations, the physical interpreted results are exhibited, analyzed and discussed. Physical parameters throughout the numerical simulation are specified in each graph. Further, the graphical results are displayed for diverse values of physical parameter in two geometries, one is the flat plate geometry $(\gamma=0)$ and other is the cylinder geometry $(\gamma=1)$.

During the numerical calculations, some dimensionless parameters values have been fixed at $\lambda_{1}=\lambda_{2}=0.1, \alpha_{1}=\alpha_{2}==0.2, \mu_{n}=0.5, \operatorname{Pr}=0.72, E c=0.5$; these numeric values are presented as universal throughout the study distant from the variations in the consequent graphs and tables. In this article, the dashed and solid lines indicate the stretching cylinder and flat plate correspondingly.

Figure 2 displays the impact of magnetic body force parameter $M n$ on velocity profile $f^{\prime}(\eta)$. In the direction of $\mathrm{x}$-axis, the transverse magnetic field (imposed in the $y$-direction, i.e. $\zeta$ - direction) produces an imminent Lorentz magneto hydro dynamic body force. This makes a major resistance to the boundary layer flow and incites a deceleration in the velocity distribution. The case $M n=0$ relates to electrically non conducting polymer flow in which magneto hydro dynamic influences vanish. Physically, the Lorentz force is increasing for increasing values of $\mu$ and it gives a significant control on the boundary layer flow and substantial retardation which might be oppressed in materials dispensation operations.

Figure 3 delineates the velocity field $f^{\prime}(\zeta)$ with the effects of

Deborah number $\lambda_{1}$ (associated with Maxwell fluid). In this diagram, we observe that the Deborah number increases when the velocity field $f^{\prime}(\zeta)$ decreases. Physically, the relaxation time is the depending factor of Deborah number. It is well known that the fluid flow resisted by higher relaxation time. In this larger Deborah number corresponds to larger relaxation time. So with this reason as the Deborah number increases, the velocity field $f^{\prime}(\zeta)$ and the momentum boundary layer thickness decrease. We also observed from figure 3 , the results with $\lambda_{1}=0$ corresponds to the viscous fluid results.

From fig. 4 that it shows that the velocity level along the cylinder or plate to be diminish by the existence of slip velocity within the boundary layer. Furthermore, at the surface of the cylinder the distribution of velocity decreases with increasing values of the slip velocity parameter. But at larger distance, the distribution of velocity is raises. Actually, when slip takes place $\left(\alpha_{1} \neq 0\right)$ the flow velocity at the cylinder will be less than the velocity of the stretching cylinder. Furthermore, increasing values of $\alpha_{1}$ decreases the flow velocity, because all the pulling forces pertaining to stretching cylinder cannot be transmitted to the fluid at the slip condition. From the figure 5 we can say that a boost in the thickness of the momentum boundary layer and velocity are affected by a raise in curvature parameter $\gamma$. When the curvature parameter attains larger value, the radius of the cylinder decreases which causes to offer less resistance by the surface increasing the fluid velocity.

The value $\lambda_{1}=0$ gives a viscous incompressible Newtonian fluid. From the figure 2 we can say that by improving Deborah number $\lambda_{1}$, the velocity distribution is observed to be decreasing. In our physics, fluid will come to rest when shear stress is eliminated This kind of phenomenon is revealed in a lot of liquid polymers that which can't be definite in the viscous fluid model. A damping force will be developed between two adjacent layers of the flow by higher abolition of Deborah number $\lambda_{1}$. By this reason there is a reduction in the velocity as well as layer thickness. When $\lambda_{1}=0$, we get the results of viscous fluid.

Figures 6-12 are plotted to evaluate the nature of the fluid temperature $\theta(\zeta)$ in opposition to the parameters $M n, \lambda_{1}, \alpha_{1}, \lambda_{2}, \alpha_{2}, E c$ and $\operatorname{Pr}$ for flat both plate and cylinder surfaces, respectively. Figure 6 show that the growth of the magnetic parameter $M n$ enhances strongly the temperature magnitudes. The supplementary word exhausted in dragging the polymer in opposition to the exploit of the magnetic field (graph 2) is dissipated as thermal energy i.e. heat. This energizes the boundary layer to increase its thickness. In the diagram the smooth profiles in the free stream (and indeed also in all other figures) indicate that a sufficiently higher value for infinity is imposed in the SRM solutions.

It is witnessed from figures 7 and 8 that by enhance in $\alpha_{1}$ and

$\lambda_{1}$ temperature of the fluid enhance all over the boundary layer region. Because the action of velocity slip factor and Deborah number has led to the decrease in the fluid velocity, therefore, extra work made in dragging the fluid in opposition to these three physical entities dissipates in the energy and hence improved fluid temperature is noticed in the boundary layer.

Fig. 9 displays that there is a noticeable shrink in temperature all over the boundary layer for higher values of non- Fourier Deborah number $\lambda_{2}$. Due to heat flux relaxation time, the associated reduction in the Deborah number $\lambda_{2}$ embodies the supplementary effect, that was captured in the non-Fourier model and that is absent in the classical Fourier model. By taking $\lambda_{2}=0$, the non- Fourier Cattaneo-Christov heat flux sculpt will be reduced to simple Fourier law of heat conduction. While the fluid in a longer heat flux is associated with lower temperature, fluids with flux relaxation time are associated with higher temperature. With growing this causes a privileged. The higher rate of heat transfer from the fluid to the wall and a lower temperature within the fluid that was caused by the increasing Deborah number $\lambda_{2}$, as a longer heat flux is 
achieved. i.e. heat is depleted from the fluid. A decrement in the thermal boundary layer thickness also one of the result. The growing temperature jump parameter $\alpha_{2}$ promotes the decrease in the temperature is clearly shown in Figure 10.

The thermal contact resistances reduces the amount of the extension of the diverse temperature jump parameters lead to diverse temperature of the cylinder is clearly stating that it is easy to understand that the temperature jump between the wall and the fluid. Furthermore, the influence of the temperature jump parameter on the temperature becomes smaller with the increasing distance from the cylinder. Figure 11 illustrated that temperature profile for flat plate and cylinder with the variation in Eckert number Ec. Evidently larger values of $E c$ lead to larger temperature. It is realistic that the dissipative heat due to elastic deformation and viscosity lead to energy storage inside the fluid region. In another way, fractional heating is the basis of heat storage in the liquid. It is noticeable from figure 12 that temperature neighboring to the wall rises and then decreases with the boost in $\mathrm{Pr}$. As Prandtl number rises, thickness of thermal boundary layer reduces. As we know that, momentum diffusivity exceeded by thermal diffusivity, i.e. heat will disseminate quickly than momentum. Temperature noticed to be squeezing nearer and nearer to wall as Pr rises. This means that fluid is extremely conductive when $\operatorname{Pr}<1$. So that heat from cylinder diffuses quicker than for large Pr fluids. So, in conducting flows, cooling rate enhances by using Prandtl number. Also, the curves are more prominent in the case of flow over a flat plate and are very close to each other for the flow over a stretching cylinder. The impact of velocity slip parameter $\alpha_{1}$ and Deborah number $\lambda_{1}$ on the local skin friction coefficient is shown in Figure 13. The rise in the slip velocity parameter $\alpha_{1}$ lead to reduce in the local skin friction coefficient is also identified. It may be observed that a decrease in the local skin friction coefficient influences an increase in Deborah number $\lambda_{1}$. Actually, the stretching velocity of the cylinder is not identical for long time near cylinder flow velocity when slip occurs, i.e. velocity slip exists. Additionally, under the slip condition, the pulling of the stretching cylinder can be only partially transmitted to the fluid. So raising values of the slip velocity will diminish the flow velocity. Figure 14 explains the effects of the temperature jump $\alpha_{2}$ and non-Fourier Deborah number $\lambda_{2}$. We observe that, as rise in parametric values of $\alpha_{2}$ wall temperature gradient is diminishing but as rise in parameter values of $\lambda_{2}$ the wall temperature is rising.

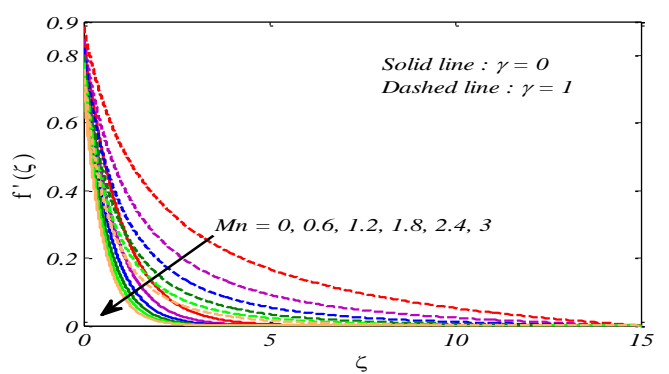

Fig. 2: Variation of Dimensionless velocity profiles $f^{\prime}(\zeta)$ with $M n$.

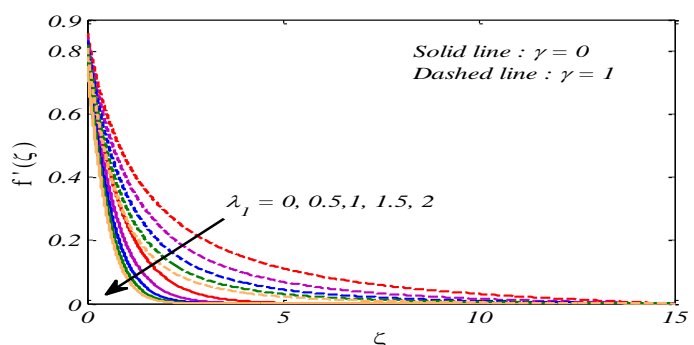

Fig. 3: Variation of Dimensionless velocity profiles $f^{\prime}(\zeta)$ with $\lambda_{1}$.

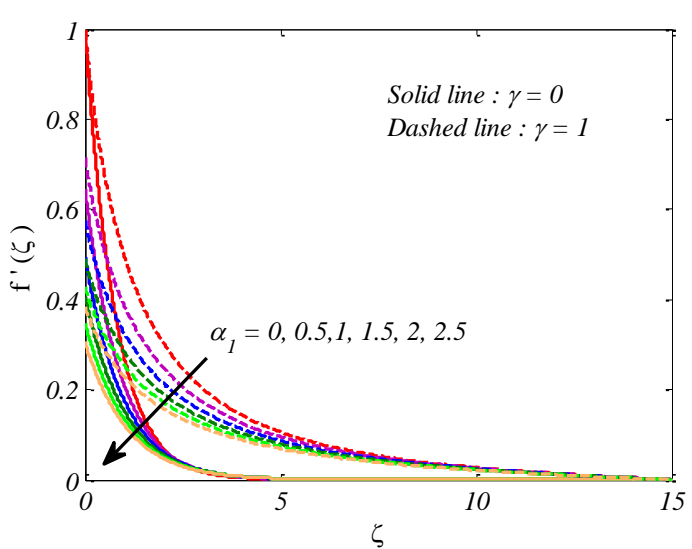

Fig. 4: Variation of Dimensionless velocity profiles $f^{\prime}(\zeta)$ with $\alpha_{1}$.

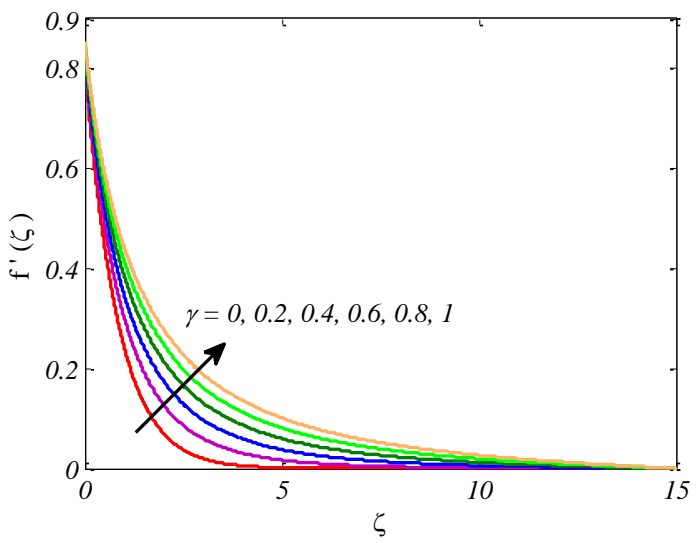

Fig. 5: Variation of Dimensionless velocity profiles $f^{\prime}(\zeta)$ with $\gamma$.

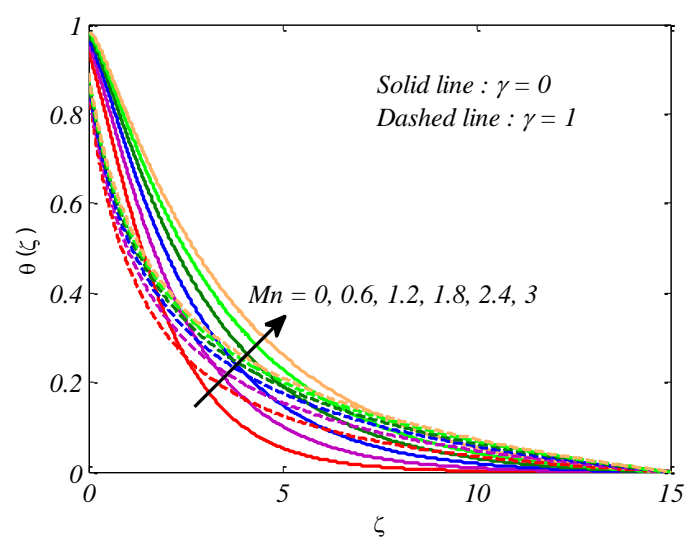

Fig. 6: Variation of Dimensionless temperature profiles $\theta(\zeta)$ with $M n$

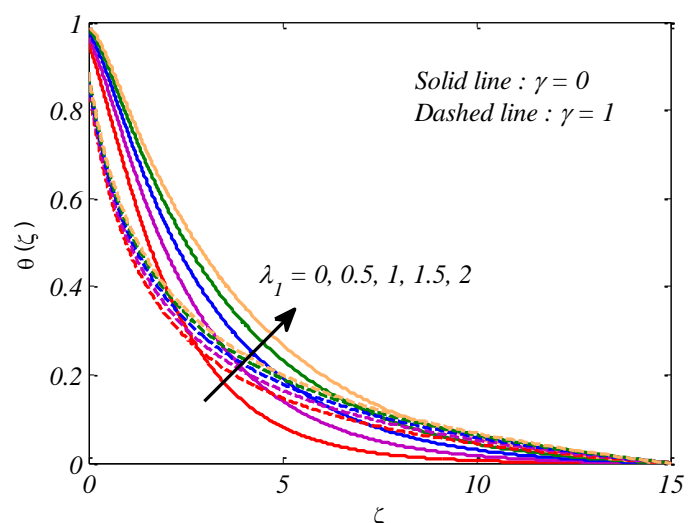

Fig. 7: Variation of Dimensionless temperature profiles $\theta(\zeta)$ with $\lambda_{1}$. 


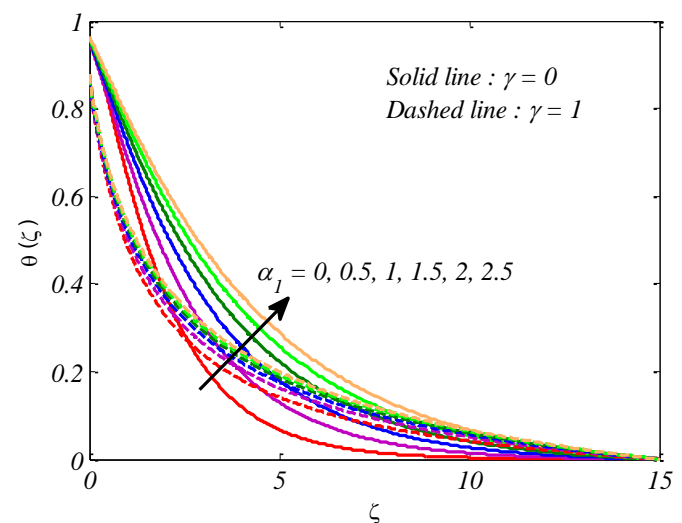

Fig. 8: variation of Dimensionless temperature profiles $\theta(\zeta)$ with $\alpha_{1}$.

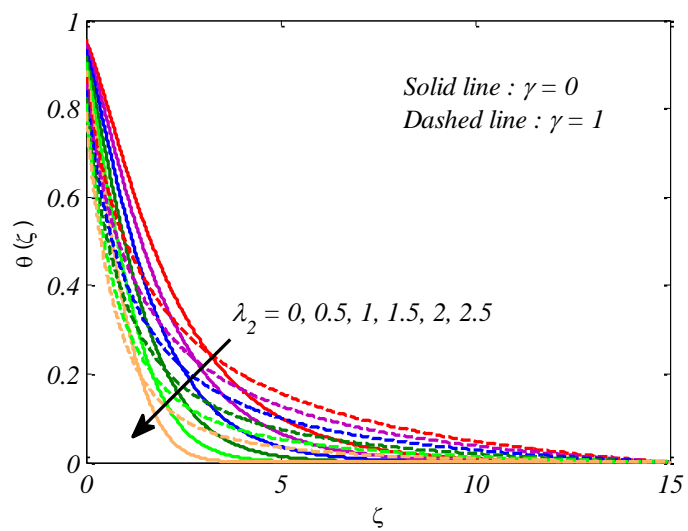

Fig. 9: Variation of Dimensionless temperature profiles $\theta(\zeta)$ with $\lambda_{2}$.

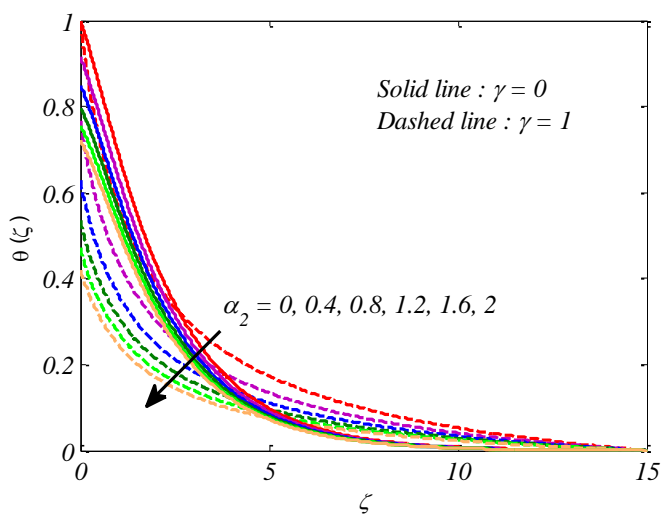

Fig. 10: Variation of Dimensionless temperature profiles $\theta(\zeta)$ with $\alpha_{2}$.

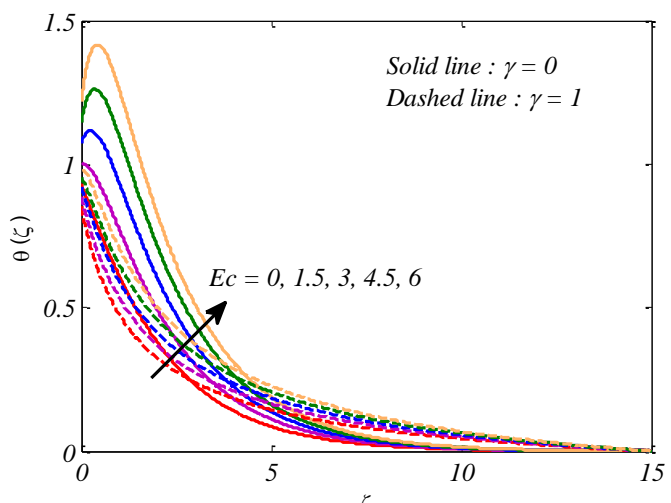

Fig. 11: Variation of Dimensionless temperature profiles $\theta(\zeta)$ with $E c$.

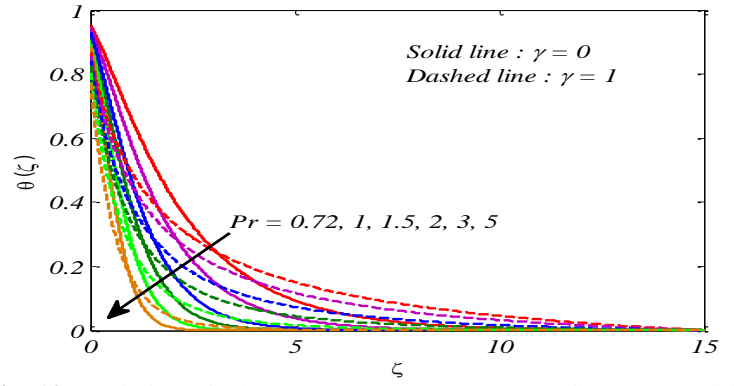

Fig. 12: Variation of Dimensionless temperature profiles $\theta(\zeta)$ with $\mathrm{Pr}$.

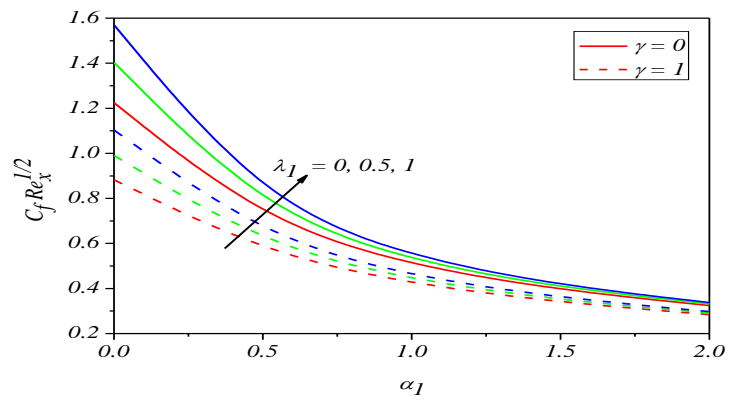

Fig. 13: Variation of Skin friction coefficient $C_{f} \operatorname{Re}_{x}^{1 / 2}$ with $\alpha_{1}$ and $\lambda_{1}$.

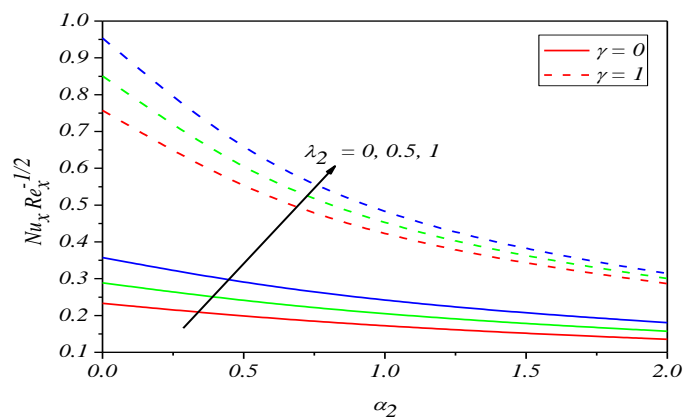

Fig. 14: Variation of local Nusselt number $N u_{x} \operatorname{Re}_{x}^{-1 / 2}$ with $\alpha_{2}$ and $\lambda_{2}$.

Table 1: Assessment of Nusselt number $-\theta^{\prime}(0)$ for various values of $\mathrm{Pr}$ when $M n=E c=\alpha_{1}=\alpha_{2}=\lambda_{1}=\lambda_{2}=0$.

\begin{tabular}{|c|c|c|c|c|c|}
\hline $\operatorname{Pr}$ & $\begin{array}{c}\text { Grubka } \\
\text { and } \\
\text { Bobba } \\
{[35]}\end{array}$ & Ali [36] & Chen[37] & $\begin{array}{c}\text { Ishak } \\
{[38]}\end{array}$ & $\begin{array}{c}\text { Present out } \\
\text { come }\end{array}$ \\
\hline 0.72 & 0.4631 & 0.4617 & 0.46315 & 0.4631 & 0.4631445612 \\
1 & 0.5820 & 0.5801 & 0.58199 & 0.5820 & 0.5819767069 \\
3 & 1.1652 & 1.1599 & 1.16523 & 1.1652 & 1.1652459519 \\
10 & 2.3080 & 1.1599 & 2.30796 & 2.3080 & 2.3080039445 \\
100 & 7.7657 & & 7.76536 & 7.7657 & 7.7656516912 \\
\hline
\end{tabular}

\section{Conclusion}

In this paper, a numerical model has been introduced to examine the influence of viscous dissipation on magneto hydro dynamic slip flow of a Maxwell fluid from a stretching cylinder. The nonFourier Cattaneo-Christov heat flux model has been implemented (the Cattaneo-Christov heat flux model can be reduced to the classical Fourier law of heat conduction when Deborah number $\lambda_{2}=0$ ). By Spectral relaxation method, the dimensionless boundary value problem with boundary conditions is solved nu- 
merically and exhibited. The major outcomes are summarized as follows.

- By rising the velocity slip and magnetic field strength, the velocity distribution diminishes.

- By raising the Deborah number of Maxwell fluid, the velocity of the fluid decreases, where as the converse behaviour is computed for the skin friction coefficient.

- A considerable increase in both thermal boundary layer thickness and temperature profile observed by raising the values of viscous dissipation and magnetic parameter.

- For higher values of Prandtl number and non-Fourier Deborah number, thermal boundary layer thickness and temperature magnitude reduces.

- Heat transfer rate of fluid reduce for high values of thermal jump parameter whereas rate of heat transfer raises within the boundary layer for high values of non-Fourier Deborah number.

- The magnitude of both the Nusselt number and skin friction of flow over a stretching cylinder $(\gamma \neq 0)$ were slight higher than the flow over a stretching flat plate $(\gamma=0)$

\section{References}

[1] Dhahir, S A (1999), On non-Newtonian flow past a cylinder in a confined flow. J. Rheol. 33, 781 doi:10.1122/1.550074

[2] Martin, M J \& Boyd, I D (2006), Momentum and heat transfer in a laminar boundary layer with slip flow. J. Thermophys. Heat Transf. 20, 710-719. doi:10.2514/1.22968

[3] Hayat, T.; Abbas, Z.; Sajid, M.: Series solution for the upperconvictedMaxwell fluid over a porous stretching plate. Phys. Lett. Sect. A Gen. At. Solid State Phys. 358, 396-403 (2006). doi:10. 1016/j.physleta.2006.04.117.

[4] Hayat, T, Abbas, Z \& Sajid, M (2009), MHD stagnation-point flow of an upper-convected Maxwell fluid over a stretching surface. Chaos Solitons Fractals 39, 840-848 (2009). doi:10.1016/j.chaos.2007. 01.067.

[5] Rashidi, M M, Beg, O A, Mehr, N F, Hosseini, A \& Gorla, R S R (2012), Homotopy simulation of axisymmetric laminar mixed convection nanofluid boundary layer flow. Theor. Appl. Mech. 39, 365-390.

[6] Shateyi, S (2013), A new numerical approach to MHD flow of a Maxwell fluid past a vertical stretching sheet in the presence of thermophoresis and chemical reaction. Bound. Value Probl. 196, 114. doi:10.1186/1687-2770-2013-196.

[7] Sajid, M, Abbas, Z, Ali, N, Javed, T \& Ahmad, I (2014), Slip flow of a Maxwell fluid past a stretching sheet. Walailak J. Sci. Technol. 11, 1093-1103.

[8] He, X \& Cai, C (2017), Near Continuum Velocity and Temperature Coupled Compressible Boundary Layer Flow over a Flat Plate, Braz J Phys 47:182-188 DOI 10.1007/s13538-017-0488-x

[9] Xinhui SI, Haozhe LI, Yanan SHEN, Liancun ZHENG, Effects of nonlinear velocity slip and temperature jump on pseudo-plastic power-law fluid over moving permeable surface in presence of magnetic field, Appl. Math. Mech. -Engl. Ed. DOI 10.1007/s10483017-2178-8

[10] Hosseini, E, Loghmani, G B, Heydari, M, \& Rashidi, M M (2017), Numerical investigation of velocity slip and temperature jump effects on unsteady flow over a stretching permeable surface, Eur. Phys. J. Plus 132: 96, DOI 10.1140/epjp/i2017-11361-8.

[11] Daniel, Y S, Aziz, Z A, Ismail, Z \& Salah, F (2017): Effects of slip and convective conditions on MHD flow of nanofluid over a porous nonlinear stretching/shrinking sheet, Australian Journal of Mechanical Engineering, DOI: 10.1080/14484846.2017.1358844.

[12] Fourier, J B J (1822), Theorie analytique de la chaleur. English translation: The analytic theory of heat, Firman Didot, Paris.

[13] Cattaneo, C (1948), Sulla conduzione del calore. Atti Semin Mat Fis della Universita` di Modena 3:3

[14] Christov, C I (2009), On frame indifferent formulation of the Maxwell-Cattaneo model of finite-speed heat conduction. Mech Res Commun 36(4), 481-486.

[15] Ostoja-Starzewski, M (2009), A derivation of the MaxwellCattaneo equation from the free energy and dissipation potentials. Int J Eng Sci 47(7), 807-810.
[16] Tibullo, V \& Zampoli, V (2011), A uniqueness result for the Cattaneo- Christov heat conduction model applied to incompressible fluids, Mech Res Commun 38(1), 77-79.

[17] Straughan, B (2010), Thermal convection with the Cattaneo- Christov model. Int J Heat Mass Transf 53(1), 95-98

[18] Haddad, S A M (2014), Thermal instability in Brinkman porous media with Cattaneo-Christov heat flux. Int J Heat Mass Transf 68, 659-668..

[19] Ciarletta, M \& Straughan, B (2010), Uniqueness and structural stability for the Cattaneo-Christov equations. Mech Res Commun 37(5), 445-447.

[20] Al-Qahtani, H \& Yilbas, B S (2010), The closed form solutions for Cattaneo and stress equations due to step input pulse heating. Phys B 405(18), 3869-3874.

[21] Papanicolaou, N C, Christov, C I \& Jordan, P M (2011), The influence of thermal relaxation on the oscillatory properties of twogradient convection in a vertical slot. Eur J Mech B/Fluids 30(1), 68-75.

[22] Han, S, Zheng, L, Li, C \& Zhang, X (2014), Coupled flow and heat transfer in viscoelastic fluid with Cattaneo-Christov heat flux model. Appl Math Lett 38:87-93

[23] Mustafa, M (2015), Cattaneo-Christov heat flux model for rotating flow and heat transfer of upper-convected Maxwell fluid. AIP Adv $5(4), 047109$.

[24] Bissell, J J (2015), On oscillatory convection with the CattaneoChristov hyperbolic heat-flow model. Proc R Soc A 471:20140845.

[25] Khan, M I, Waqas, M, Hayat, T, Khan, M I \& Alsaedi, A Chemically reactive flow of upper-convected Maxwell fluid with Cattaneo-Christov heat flux model, J Braz. Soc. Mech. Sci. Eng. DOI 10.1007/s40430-017-0915-5.

[26] Raju, C S K, Sanjeevi, P, Raju, M C, Ibrahim, S M, Lorenzini, G, \& Lorenzini, E, The flow of magnetohydrodynamic Maxwell nanofluid over a cylinder with Cattaneo-Christov heat flux model, Continuum Mech. Thermodyn. DOI 10.1007/s00161-017-0580-z.

[27] Shahid, A, Bhatti, M M, Anwar, Be'g, O \& Kadir, A, Numerical study of radiative Maxwell viscoelastic magnetized flow from a stretching permeable sheet with the Cattaneo-Christov heat flux model, Neural Comput \& Applic, DOI 10.1007/s00521-017-2933-8.

[28] Khana, S M, Hammad, M, Batool, S, \& Kaneez, H, (2017), Investigation of MHD effects and heat transfer for the upper-convected Maxwell (UCM-M) micropolar fluid with Joule heating and thermal radiation using a hyperbolic heat flux equation, Eur. Phys. J. Plus 132: 158, DOI 10.1140/epjp/i2017-11428-6

[29] Sheikholeslami, M \& Bhatti, M M (2017), Active method for nanofluid heat transfer enhancement by means of EHD. Int J Heat Mass Transf 109, 115-122.

[30] Ocone,, R, Astarita G (1987), Continuous and discontinuous models for transport phenomena in polymers. AIChemE J 33, 423-435

[31] Zhe, Z \& Dengying, L (2000), The research progress of the nonFourier heat conduction. Adv Mech 30,123-141.

[32] Huilgol, R R, (1992), A theoretical and numerical study of nonFourier effects in viscometric and extensional flow of an incompressible simple fluid. $J$ Non-Newtonian Fluid Mech 43,83-102.

[33] Motsa, S S \& Makukula, Z G (2013), On spectral relaxation method approach for steady von kárman flow of a reiner-rivlin fluid with joule heating, viscous dissipation and suction/injection. Cent. Eur. J. Phys., 11(3), 363-374.

[34] Kameswaran, P, Sibanda, P, \& Motsa, S S, (2013), A spectral relaxation method for thermal dispersion and radiation effects in a nanofluid flow. Boundary Value Problems 2013, 242.

[35] Grubka, L J, Bobba, K M (1985), Heat transfer characteristics of a continuous, stretching surface with variable temperature. ASME J Heat Transf 107, 248-25.

[36] Ali, M E, (1994), Heat transfer characteristics of a continuous stretching surface. Heat Mass Transfer 29, 227-234

[37] Chen, C H (1998), Laminar mixed convection adjacent to vertical, continuously stretching sheets. Heat Mass Transfer, 33,471-476.

[38] Anuar Ishak (2010), Thermal boundary layer flow over a stretching sheet in a micropolar fluid with radiation effect, Meccanica 45 , 367-373, DOI 10.1007/s11012-009-9257-4 